\title{
TRASTORNO DE PERSONALIDAD: LA MIRADA DIMENSIONAL del Diagnóstico Psicodinámico Operacionalizado (OPD-2)
}

\author{
Personality Disorder: The Dimensional View of the \\ Operationalized Psychodynamic Diagnosis (OPD-2).
}

\section{Guillermo de la Parra-Cieciwa ${ }^{1}$, Jorge Humberto Vanegas-Osorio ${ }^{2}$}

doi: https://doi.org/10.17533/udea.rp.e343219

\section{Resumen}

La mirada categorial de los trastornos de personalidad proviene del modelo médico donde la enfermedad mental es vista como una condición discreta, con claros límites entre salud y enfermedad. Esta perspectiva fue adoptada por las diferentes clasificaciones modernas como el DSM- 4 y el CIE- 10 . Recientemente se ha cuestionado aquella mirada categorial de los trastornos de personalidad, de tal modo que la clasificación CIE-11 y la sección III del DSM-5 han adoptado una perspectiva dimensional, aunque este último mantuvo un sistema de categorías de los trastornos de personalidad en su clasificación oficial. Un aporte que se adelantó a este cambio de paradigma es el Diagnóstico Psicodinámico

\section{Abstract}

The categorical view of personality disorders comes from the medical model where men-

Recibido: 2020-07-08 / Aceptado: 2020-12-22

Para citar este artículo en APA: Parra-Cieciwa, G. de la y Vanegas-Osorio, J. (2020). Trastorno de la personalidad: la mirada dimensional del diagnós-

tico psicodinámico operacionalizado (OPD-2).

Revista de Psicología de la Universidad de Antioquia, 12(2), e343219. doi: https://doi.org/10.17533/ udea.rp.e343219
Operacionalizado (OPD-2), sistema de diagnóstico desarrollado en Alemania en los ańos noventa, que permite una evaluación clínica y terapéuticamente relevante de los patrones relacionales disfuncionales del consultante, de sus conflictos principales y de las disfunciones de personalidad desde una perspectiva dimensional. Se concluye que el opD-2 es una contribución significativa contemporánea a la comprensión y el manejo de los así llamados trastornos (disfunciones) de personalidad.

Palabras clave: trastorno de personalidad, estructura de personalidad, diagnóstico psicodinámico, opD-2.

tal illness is seen as a discrete condition, with clear limits between health and illness. This

1 Doctor en Medicina, Universidad de Ulm, Alemania. Médico Psiquiatra, Unidad de Psicoterapia de Adultos, Facultad de Medicina, Departamento de Psiquiatría, Pontificia Universidad Católica de Chile. Correo: gdelaparra@uc.cl; https://orcid.org/0000-0002-3498-3012.

2 Doctor en Psicología Clínica y de la Salud. Profesor Departamento de Psicología de la Universidad de Antioquia; https://orcid.org/00000002- 8223-6660. 
perspective was adopted by the different modern classifications such as the DSM- 4 and the CIE-10. This categorical view of personality disorders has recently been questioned, in such a way that the ICD-11 classification and section III of the DSM-5 have taken a dimensional perspective, although the latter maintained a system of categories of personality disorders in its official classification. A contribution that came before this paradigm shift is the Operationalized Psychodynamic Diagnosis (OPD-2), a diagnostic system deve- loped in Germany in the 1990s, which allows a clinically and therapeutically relevant evaluation of the patient's dysfunctional relational patterns, major conflicts and personality dysfunctions from a dimensional perspective. It is concluded that OPD- 2 is a significant contemporary contribution to the understanding and management of so-called personality disorders (dysfunctions).

Keywords: personality disorder, personality structure, psychodynamic diagnosis, opD-2.

\section{Introducción}

El punto de partida para el desarrollo de este artículo sobre la mirada dimensional de los trastornos de la personalidad (тр) es presentar el sistema Diagnóstico Psicodinámico Operacionalizado (OPD) que le sirve de soporte teórico y de aplicación clínica.

El opD-2 fue desarrollado en Alemania por un grupo de trabajo conformado por psicoanalistas especializados en medicina psicosomática y psiquiatría. Surge de la creciente disconformidad con los sistemas clasificatorios descriptivos CIE y DSM y de la dificultad de comunicación entre profesionales de las diferentes orientaciones teóricas dentro del psicoanálisis.

El opd-2 es un instrumento relevante clínicamente y se caracteriza por su utilidad básica al mantener las formulaciones en un nivel de abstracción medio, entre una descripción conductual pura y una formulación puramente metapsicológica, por lo que ha contribuido a mejorar la comunicación dentro de la comunidad clínica. Este sistema de diagnóstico se ha implementado como un recurso para investigación científica, con criterios rigurosos para elegir muestras y lograr mayor homogenización en los estudios aleatorios. Permite evaluar fenomenológica y psicodinámicamente el curso de la enfermedad y facilita la revisión de indicaciones y directrices alternativas, la determinación de patrones individuales en diferentes áreas de la vida y la investigación de la efectividad de la psicoterapia.

El sistema se compone de cuatro ejes psicodinámicos y de un eje descriptivo para la comprensión clínica de los problemas de la salud mental de un indi- 
viduo. Permite identificar los patrones relacionales disfuncionales, las constelaciones conflictivas intrapsíquicas y las condiciones estructurales del paciente. Con base en los ejes es posible deducir los focos terapéuticos. Además, las categorías OPD-2 identificadas permiten observar y evaluar los cambios inducidos por la psicoterapia.

La explicación y el significado que el paciente aporta acerca de sus problemas, sumado a las hipótesis e inferencias psicodinámicas que el terapeuta formula sobre la base de constructos cercanos a lo observable - por tanto operacionalizables-, permiten el establecimiento de los focos de trabajo psicoterapéutico, que se refieren a las áreas problemáticas que están en la base de los síntomas psíquicos o psicosomáticos, las disfunciones relacionales, los conflictos intrapsíquicos y las vulnerabilidades estructurales: "El foco terapéutico no es algo externo, ofrecido y prescrito para el paciente, sino que está contenido en el material que él ofrece" (OPD-2, 2008, p. 406)

Esta ruta diagnóstica permite al terapeuta identificar en su paciente los núcleos conscientes e inconscientes centrales del trastorno clínico; es así como la integración de los focos OPD-2, facilitados por la operacionalización de los ejes, y con base en hipótesis psicodinámicas hechas "a la medida" para cada paciente en particular, se pueden condensar en una formulación psicodinámica de caso y servirse de ella como directriz diagnóstica con el fin de establecer la indicación del tratamiento, la planificación del proceso y la evaluación del cambio terapéutico. Un progreso psicoterapéutico importante se alcanzará sólo si existen cambios en el foco.

Validez y confiablidad: en el manual opD-2 (pp. 62-73) se presentan y se discuten las investigaciones de validez y confiabilidad sobre_cada uno de los ejes y desde su publicación se han dado a conocer numerosos estudios acerca de sus atributos clínicos y empíricos. (Thomasius et al., 2001; Cierpka et al., 2006; Cierpka et al, 2010). Las revisiones sistemáticas, evaluativas y clínicas exponen las evidencias sobre una fiabilidad aceptable para fines clínicos e investigativos reproducibles. Los estudios de validez indican además el alto nivel de congruencia interna y externa del opD-2 como una herramienta clínica esencial para la comprensión dimensional de los trastornos de la personalidad y para la investigación científica con diferentes unidades 
de estudio en diversos ámbitos ambulatorios y hospitalarios (Both, Bastos y Freitas, 2019).

Puesto que el opD-2 desarrolla un sistema modular para el diagnóstico y la planificación del tratamiento ha sido empleado en diferentes ámbitos del quehacer clínico y de la investigación empírica y, con ello, los estudios sobre sus índices de validez y confiabilidad en cada eje o módulo, según el contexto de aplicación. Por ejemplo, Waage et al. (2011) muestran validez en la estructura interna, así como la aceptabilidad de la eficacia de los factores subyacentes del eje I, y años atrás Schneider et al. (2000) habían reportado las propiedades psicométricas y el papel de la defensa y las teorías de afrontamiento en la conceptualización del eje I, así como la contribución de los ítems de este eje a la predicción de los resultados del tratamiento.

Sobre la validación del eje II, Grande et al. (2005) concluyen que el eje relación basado en el modelo circunflejo de la conducta interpersonal es adecuado para una gama más amplia de métodos de evaluación vinculados a este sistema y contiene, además, una guía que indica cómo formular un resumen de la dinámica de las relaciones. Por lo tanto, los resultados de diagnósticos pueden resumirse con fines clínicos prácticos y usarse directamente en el trabajo terapéutico. Este diagnóstico relacional del opD-2 (eje II) se basa en el modelo circunflejo de conducta interpersonal, el cual tiene una larga tradición en psicología de la personalidad, social y clínica, y, por lo tanto, ha sido validado.

A propósito de los criterios de validez del opD-2, Schneider et al. (2008) compararon los criterios de operacionalización que este manual sugiere del cuerpo y la sexualidad con la autoevaluación de la escala de concepto corporal de Frankfurt.

. Con una muestra de 105 pacientes en psicoterapia fue posible evaluar las hipótesis psicodinámicas respecto a conflictos y a los niveles de estructura. En la misma línea, Pieh et al. (2009) en su ensayo clínico se ocupan de controlar la validez de los conflictos inconscientes dentro del OPD-2, verificando las hipótesis mediante análisis psicométricos; asimismo, Doering et al. (2014) concluyen en su estudio que el eje III muestra un índice adecuado de fiabilidad y de validez concurrente y discriminante y puede, por tanto, ser recomendado para uso clínico y de investigación. 
Respecto al eje IV del opd-2 (estructura), Spitzer et al. (2002) publican los hallazgos sobre la validez satisfactoria de este eje. Más adelante, Zimmermann et al. (2012) vinculan el nivel de integración estructural del sistema de diagnóstico psicodinámico operacionalizado (OPD-2) con el nivel de funcionamiento estructural de la sección III del DSM-5; más precisamente, estos investigadores presentan una discusión conceptual y empírica de los niveles de escala de funcionamiento de la personalidad desde la perspectiva del OPD-2 frente a las propuestas de la evaluación de la gravedad global de deterioro en el funcionamiento de la personalidad en el DSM- 5 .

\section{Cambio de paradigma en trastornos de personalidad}

En la última década se ha incrementado el consenso entre los psicoterapeutas y los psiquiatras clínicos sobre la poca utilidad de los sistemas categoriales para orientar la intervención del paciente. A continuación, serán presentados algunos ejemplos de este cambio de paradigma. Haslam, Holland y Kuppens (2012) sostienen que "La presunción de que es posible diferenciar categorialmente a personas con y sin trastornos de personalidad, se ha demostrado como empíricamente insostenible" (excepto para TP esquizotípico) (p. 25). La APA (2000) reconoce que: "los trastornos de personalidad representan variantes maladaptativas de rasgos de personalidad que se continúa imperceptiblemente con la normalidad" (p. \#). Es así como el DSM-5 reconoce explícitamente la continuidad de los trastornos mentales, la imposibilidad de definir categorías discontinuas y discretas: "no hay límites naturales entre los síndromes mayores, lo que hace deficiente el enfoque categorial: los límites entre trastornos son más porosos de lo que se pensaba”. (Echevarría-Pérez 2015, p. 689).

$\mathrm{Al}$ respecto, Fontenelle et al. (2011) sustentan que el trastorno obsesivo compulsivo (TOC), los trastornos de control de impulsos (ICD) y los trastornos relacionados con el consumo de sustancias (SUD) se superponen en diferentes niveles, incluyendo fenomenología, comorbilidad, neurocircuito, neurocognición, neuroquímica e historia familiar.

Los numerosos análisis factoriales muestran que el ordenamiento de las características (mezcla de rasgos y síntomas) en las diversas categorías no se 
agrupa según lo esperado, sino que se distribuyen a través de los diferentes diagnósticos, lo que redunda en que los pacientes frecuentemente cumplen con los criterios de más de un diagnóstico o comorbilidad artificial (Trull y Durret, 2005). Las nominaciones comorbilidad, concurrencia y diagnóstico dual, son formas de contener la fragilidad valorativa que afecta su validez. La comorbilidad psiquiátrica parece ser la regla más que la excepción, a saber: los diagnósticos simultanéanos entre fobia social, ansiedad generalizada y ansiedad por separación; estos, a la vez, muy asociados a la depresión. Es así que pacientes con problemas muy distintos pueden caer en la misma categoría (gran heterogeneidad en una consideración diagnóstica); a la vez, indicadores como impulsividad, agresividad, suicidio, ansiedad y depresión pueden aparecer como propios de diagnósticos diversos. Por último, muchos pacientes describen un patrón de dificultades específicas que no permite ser clasificado dentro un rango común (prevalencia significativa de TP no especificado).

La evidencia apoya la existencia de un continuo entre personalidad normal y anormal (Haslam, Holland y Kuppens, 2012). Las descripciones basadas en rasgos predicen más acertadamente la disfunción a largo plazo que las categorías de Tp. Otra característica es que el individuo queda descrito por la ubicación que tiene en cada una de las dimensiones y no por la pertenencia a una categoría concreta. Por ejemplo, un mismo paciente puede tener un grado de depresión, uno de ansiedad y otro de impulsividad (Echevarría-Pérez, 2015).

En el DSM-5 la definición de diagnóstico de trastorno de personalidad no ha cambiado con respecto a la del DSM-4-TR. La implementación de un modelo dimensional fue bloqueada en una intervención de último minuto por un sector de la misma Asociación Americana de Psiquiatría, con el argumento de que se perdería información valiosa del DSM-4 y que los psiquiatras no lograrían adaptarse al nuevo sistema. Sin embargo, se incluyó como apéndice un modelo alternativo (Sección III del DSM-5) para diagnosticar trastornos de la personalidad en el que se plantea un continuo según el funcionamiento del self (identidad) y de las relaciones interpersonales (relaciones objetales) de acuerdo con el Level of Personality Functioning Scale (LPFS) (Bender, Morey y Skodol, 2011; Morey et al., 2011), manteniendo categorías según los rasgos patoló- 
gicos. Con este enfoque, el nuevo sistema de clasificación ofrecería, tanto a clínicos como a investigadores, la oportunidad de describir al paciente con mucho más detalle que antes. Esencialmente, el comité que formuló la sección III (Modelos y Medidas Emergentes), propuso un modelo híbrido de TP, es decir, categórico y dimensional; uno que combina la noción de categorías discretas con calificaciones dimensionales suplementarias de rasgos de personalidad relevantes. Estudios longitudinales muestran que, si bien los pacientes pueden remitir respecto a su trastorno a través del tiempo, no cumpliendo con los criterios de TP, continúan registrándose dificultades funcionales como una característica permanente en mucho de ellos (Herpetz et al., 2017).

El CIE-11, se diferencia del DSM-5 con nuevo enfoque dimensional. El CIE 11 abolió las categorías de trastorno de personalidad, excepto una: hay presencia o ausencia de trastorno de personalidad de acuerdo con un continuo desde "dificultades de la personalidad" hasta trastorno leve, moderado o grave. Propone una clasificación amplia con base en: 1) los criterios generales de un trastorno de personalidad; 2) la identificación de los niveles de severidad; y 3) la evaluación según cinco dominios de rasgos (de afecto negativo, disocial, de desinhibición, anancástico y de desapego). Plantea, entonces, que las anormalidades de la personalidad son un set de constructos dimensionales: desregulación emocional vs. estabilidad, extraversión vs. introversión, antagonismo vs. docilidad (conformidad), impulsividad vs. represión. La ubicación dentro del continuo se realiza básicamente de acuerdo con descriptores interpersonales.

Muchos psiquiatras prefieren los sistemas categoriales a los dimensionales porque las decisiones clínicas a menudo también son categoriales (por ejemplo, diagnosticar o no, tratar o no). Sin embargo, los sistemas dimensionales se pueden convertir en categoriales, como en el caso del CIE-11, para satisfacer las necesidades clasificatorias de las instituciones o del clínico, siempre que estén disponibles los límites apropiados, así como los algoritmos de decisión. Además, en la medida en que los modelos dimensionales brinden una descripción confiable y válida de los pacientes, los médicos y los investigadores, preferirían estos procedimientos evaluativos. Esta visión de la psicopatología y de la concepción de la organización psíquica se alinea 
notablemente con las avanzadas teorías e investigaciones psicodinámicas. El CIE 11 y el modelo alternativo de TP en el DSM-5 (sección III) representan un paso en dirección a la adopción de nosologías impulsadas cualitativa y empíricamente. Es por esto que, sumado a la evidencia preliminar de utilidad, los enfoques dimensionales se han vuelto cada vez más recurrentes en la literatura y se han comenzado a integrar sus evaluaciones (Henkel et al, 2018; Gøtzsche-Astrup y Moskowitz, 2016).

\section{Dimensiones psicodinámicas básicas: ejes OPD-2}

El sistema OPD-2 se adelantó notoriamente a los sistemas dimensionales descritos más arriba (se creó en su primera versión a principios de los noventa) al plantear la personalidad como un conjunto de capacidades o funciones, que podían evaluarse independientemente en un continuo dimensional de mayor a menor nivel de integración (véase más adelante). A continuación, se describen las características del sistema OPD-2 para, luego, detallar cómo entiende el funcionamiento de la estructura de personalidad. Después de caracterizar los trastornos basados en conflicto y estructura, se presenta un breve caso clínico con el fin de ilustrar su aplicación.

El diagnóstico personalizado propuesto por el opD-2 estima los datos del paciente como componentes de procesos dinámicos contextualizados, que son únicos para cada individuo. A continuación, se presenta una reseña de los indicadores de evaluación de los ejes.

\section{Eje I: la experiencia de enfermedad o problema y prerrequisitos del trata- miento}

Abarca 19 indicadores que representan dimensiones para guiar la indicación terapéutica. Incluyen el rango de experiencias con la enfermedad, la fluctuación temporal de los síntomas, el modo en que se desencadena el padecimiento, los recursos y los obstáculos (internos y externos) para afrontar las dolencias, la calidad, uso de la red de apoyo de la que dispone y las expectativas relativas al tratamiento. 


\section{Eje II: la / relacional disfuncional}

Se refiere a la constelación específica que se produce en las interacciones habituales del paciente y las formas en que reaccionan sus compañeros sociales Las unidades analíticas que se evalúan en este eje son cuatro posiciones interpersonales: 1) cómo se vivencia el paciente a sí mismo; 2) cómo él vivencia a otros; 3 ) cómo otros vivencian repetidamente al paciente (aborda los aspectos inconscientes de la relación, oferta relacional inconsciente que perpetúa el problema); y, por último, 4) cómo los otros se vivencian repetidamente a sí mismo frente al paciente (diagnóstico contratransferencial).

\section{Eje III: conflicto}

Se pueden distinguir siete conflictos básicos y sus modalidades de elaboración activa o pasiva: 1) individuación versus dependencia (afecto guía: angustia existencial por fusión o abandono); 2) sumisión vs. control (afecto guía: rabia); 3) deseo de protección y cuidado vs autosuficiencia (afecto guía: tristeza, añoranza); 4) conflicto de autovaloración (afecto guía: vergüenza y humillación); 5) conflicto de culpa (afecto guía: culpa); 6) conflicto edípico (afecto guía: inhibición, rivalidad de género); y, 7) conflicto de identidad (conflicto de roles).

El concepto psicodinámico de conflicto se basa en el supuesto básico de que la conducta humana está constantemente influida por deseos, pensamientos, valores y representaciones inconscientes. La oposición de grupos motivacionales, mantenidos como trasfondo durante largo tiempo, lleva a un elevado estado de tensión interna. El modelo dimensional continuo permite para el diagnóstico del conflicto, diferenciar entre tensiones conflictivas normales y los conflictos clínicamente relevantes en una graduación continua. Es necesario distinguir entre la tensión más o menos consciente producida por los desafíos personales y aquella derivada de dificultades inconscientes.

El opD-2 evalúa cada conflicto según cómo se reproduce en diferentes contextos de funcionamiento, tales como familia de origen, pareja, trabajo y ámbitos sociales. Cada conflicto se expresa con un afecto guía bien delimitado (por ejemplo, la angustia existencial en el conflicto de autonomía versus dependencia). 


\section{Eje IV: estructura}

La estructura se refiere al tejido de disposiciones psíquicas, que abarca todo lo que en la vivencia y conducta del individuo se desarrolla de manera regular y repetitiva (consciente o alejado de la conciencia). La estructura no es rígida ni inmutable, ya que evidencia procesos de desarrollo durante toda la vida, enlazándose en ese sentido con términos como identidad, carácter o personalidad. En contraposición con la concepción estática, el enfoque dinámico entiende las estructuras psíquicas desde una perspectiva evolutiva, capaz de restaurar sus equilibrios intrapsíquicos e interpersonales.

El concepto teórico de este eje del opD-2, centra su análisis en la capacidad y vulnerabilidad del self en su relación con los otros. Se trata de la construcción de la representación intrapsíquica del mundo objetal externo (relaciones objetales), así como de las experiencias del self en el contacto con el mundo objetal (representación del selfy de su interacción con los objetos internalizados). La estructura individual no sólo se manifiesta a través de limitaciones y debilidades, sino también con sus recursos y fortalezas. Con base en esta dinámica self-objeto, el sistema OPD-2 presenta las dimensiones de la evaluación estructural (Tabla 1).

Tabla 1.

Dimensiones de la evaluación estructural

\begin{tabular}{lll}
\hline Dominio & Función & Subfunción \\
\hline 1. Capacidades cognitivas & 1a. Percepción de sí mis- & 1.1 Autorreflexión \\
& mo & 1.2 Diferenciación afectiva \\
& & 1.3 Identidad \\
& 1b. Percepción del objeto & 1.4 Diferenciación self-objeto \\
& & 1.5 Percepción de objeto total \\
& & 1.6 Percepción realista del objeto \\
2. Capacidad de manejo & 2a. Autorregulación & 2.1 Manejo de impulsos \\
& & 2.2 Tolerancia afectiva \\
& & 2.3 Regulación de la autoestima
\end{tabular}

2b. Regulación de la rela- 2.4 Protección de las relaciones

ción con el objeto $\quad 2.5$ Regulación de los intereses

2.6 Anticipación 
Trastorno de personalidad: la mirada dimensional del Diagnóstico Psicodinámico Operacionalizado (OPD-2)

3. Capacidad emocional 3a. Comunicación emo- 3.1 Vivencia de los afectos cional interna

3.2 Uso de fantasías

3.3 Self corporal

3b. Comunicación emo- 3.4 Establecer contacto cional externa

3.5 Comunicación de los afectos

3.6 Empatía

4. Capacidad de vínculo

4a. Vínculo con objetos 4.1 Internalización

internos

4.2 Uso de introyectos

4.3 Variedad de vínculos

4b. Vínculo con objetos 4.4 Capacidad de vincularse

externos

4.5 Aceptar ayuda

4.6 Desprenderse de vínculos, separarse

\section{Limitaciones estructurales}

Con el fin de responder al propósito de este artículo, se reprodujeron algunos puntos de abordaje sobre la mirada dimensional de los trastornos de la personalidad desde el opD-2, y se vincularon fragmentos ricamente argumentados en el manual de referencia (OPD-2, 2008) que permitan orientar a los lectores acerca de su contenido.

Las limitaciones estructurales también corresponden a un modelo dimensional, y se expresan en un continuo que va desde el funcionamiento normal hasta las carencias severas. La normalidad en los adultos está dada por la maduración psíquica manifestada en crecientes procesos de cohesión del self, diferenciación e integración de las relaciones objetales, cuyo resultado es la autonomía de sí-mismo con un sentido de identidad capaz de regular la autoimagen y la autoestima, así como la propia conducta y el manejo en el establecimiento de vínculos interpersonales satisfactorios.

Para marcar el alcance y la calidad del desorden o vulnerabilidad estructural, el OPD-2 distingue cuatro niveles de integración (alto, medio, bajo y desintegrado). El nivel alto de integración significa que un self autónomo tiene acceso a un espacio intrapsíquico dentro del cual se pueden elaborar los conflictos, la angustia central correspondería a la pérdida del amor del objeto. En el nivel medio de integración la persona tiene un menor acceso a funciones 
reguladoras y se reconoce una diferenciación más débil de las subestructuras psíquicas, la angustia central correspondería a la pérdida del objeto. El nivel bajo de integración se caracteriza por un escaso desarrollo del mundo intrapsíquico, de manera que los conflictos se elaboran poco internamente y más bien se manifiestan en forma de conflictos interpersonales en la realidad externa, la angustia central correspondería a la destrucción del self por el objeto malo o por la pérdida del objeto bueno. El nivel desintegrado se caracteriza por la fragmentación y por la restitución psicótica. Este último se refiere a la permanente limitación de los dominios estructurales, y la angustia central correspondería al temor por la disolución del self a causa de objetos execrables.

Tabla 2.

Niveles de Integración Estructural

\begin{tabular}{|c|c|c|c|}
\hline $\begin{array}{l}\text { Nivel alto de } \\
\text { integración }\end{array}$ & $\begin{array}{l}\text { Nivel moderado de } \\
\text { integración }\end{array}$ & $\begin{array}{l}\text { Nivel bajo de } \\
\text { integración }\end{array}$ & Nivel desintegrado \\
\hline $\begin{array}{l}\text { Un sí mismo autóno- } \\
\text { mo, adecuadamente } \\
\text { regulado, con acceso a } \\
\text { un espacio psíquico en } \\
\text { el que pueden elabo- } \\
\text { rarse los conflictos. } \\
\text { (Polo de la disponibi- } \\
\text { lidad segura de funcio- } \\
\text { nes estructurales) }\end{array}$ & $\begin{array}{l}\text { El sí mismo tiene me- } \\
\text { nor capacidad regu- } \\
\text { ladora. Es más débil } \\
\text { la diferenciación de } \\
\text { estructuras psíquicas } \\
\text { self-objeto. }\end{array}$ & $\begin{array}{l}\text { Escaso desarrollo del } \\
\text { espacio intrapsíquico, } \\
\text { escaso acceso a capa- } \\
\text { cidades de regulación. } \\
\text { Los conflictos no } \\
\text { pueden elaborarse in- } \\
\text { ternamente sino en el } \\
\text { espacio interpersonal. }\end{array}$ & $\begin{array}{l}\text { Fragmentación del } \\
\text { self. Mínimo o nulo } \\
\text { acceso a capacidades } \\
\text { regulatorias. Inundado } \\
\text { por afectos intensos y } \\
\text { desestructurados que } \\
\text { no puede verbalizar. } \\
\text { (Polo de las graves } \\
\text { limitaciones estructu- } \\
\text { rales) }\end{array}$ \\
\hline
\end{tabular}

El trastorno estructural se puede entender en el sentido de un déficit del desarrollo, que alude a capacidades estructurales que no lograron desarrollarse. El self, en consecuencia, no alcanza autonomía, es incapaz de regularse suficientemente, y no logra reflejarse a sí mismo; es decir, no pudo desarrollar un vínculo seguro con otros significativos capaces de brindar soporte. En el caso de vulnerabilidad estructural, sí hubo un desarrollo de la estructura, pero no se logró una suficiente estabilidad, lo que en situaciones de estrés interno o externo hace que se pierdan funciones ancladas estructuralmente y se activen estados de ansiedad y desintegración. Para el diagnóstico diferencial es rele- 
vante la distinción entre las capacidades estructurales habituales y manifestaciones agudas que aparecen en condiciones de estrés. Conflictos reales que ejercen una fuerte presión emocional (por ejemplo, crisis interpersonales, separaciones o sucesos vitales estresantes) pueden, en combinación con falta de sueño, grandes esfuerzos corporales, excesivo uso de alcohol, drogas, etcétera, desencadenar procesos regresivos, que se expresan como si fueran alteraciones estructurales (por ejemplo, pérdida de la regulación afectiva y de la autoestima, dudas sobre la propia identidad, rupturas en la comunicación, confusión proyectiva entre self y objeto, etcétera). En el nivel de estructura del opD-2 no se evalúan estas reacciones por estrés y los desequilibrios estructurales desencadenados eventualmente por ellas. Más bien, se busca evaluar el modo habitual y estable de funcionamiento del último año o de los últimos dos años (OPD-2, 2008, cf. pp. 141-142).

Dependiendo de la magnitud de las limitaciones condicionadas por el conflicto o por la estructura, se pueden diferenciar: trastornos basados predominantemente en conflictos y trastornos predominantemente estructurales.

El proceso de recolección e integración diagnóstica requiere de un manejo básico de los constructos psicodinámicos, de entrenamiento en la aplicación de la entrevista OpD-2 con el fin de adquirir la capacidad para recopilar los datos apropiados y la implementación de la operacionalización, además, "es deseable que exista una experiencia terapéutica personal, para poder alternar entre la observación del paciente y la auto observación de la contratransferencia” (opD-2, p. 143).

\section{Trastornos basados predominantemente en conflictos}

Ya que el conflicto y la estructura representan los polos de una complementariedad clínica, se comportan entonces como figura y fondo o semejantes a una pieza teatral (conflicto), representada en un escenario (estructura): el escenario es, pues, prerrequisito para la representación (opD-2, p. 118)

Las asociaciones y sobreposiciones entre los ejes reflejan características de la personalidad que se mueven juntas o independientemente, revelando así el funcionamiento estructural de un individuo. En este sentido se entiende que 
la estructura psíquica en cierto modo representa el fondo sobre el cual ocurren los conflictos, con sus patrones de solución, adaptativos o no (Cierpka, 2010, p. 6). Para establecer el diagnóstico del conflicto debe existir una estructura suficientemente integrada, un espacio mental que sea capaz de "contener" el conflicto. Si no, aparecerán manifestaciones que asemejarán un conflicto (esbozo de conflicto), pero que son muestra de vulnerabilidades estructurales. Por su parte, "los patrones relacionales disfuncionales pueden ser entendidos como la expresión de conflictos internos, características estructurales y al mismo tiempo de su manejo" (Cierpka, 2010, p. 227).

En estos trastornos, los procesos mentales ocurren sobre la base de un nivel de integración estructural alto o medio. La tarea consiste en formular una hipótesis acerca de cuál de los conflictos representa el núcleo del trastorno, y en determinar cuáles derivan de este conflicto o están asociados a él. Como ya se señaló en el presente artículo, es frecuente el entrelazamiento de conflictos. El OPD-2 permite un análisis detallado de estas interacciones, así como el esclarecimiento de complejos cuadros clínicos, determinando capas diferenciadas de conflictos más sencillos. En trastornos basados claramente en conflictos puede ocurrir que dos o tres conflictos sean suficientes para describir la perturbación y los focos estructurales se hagan relativamente irrelevantes.

\section{Alteraciones predominantemente estructurales}

El trastorno está determinado en gran medida por la limitada disponibilidad de funciones estructurales, como la capacidad de autorreflexión y diferenciación afectiva, la percepción realista del objeto y la empatía, autorregulación y defensas maduras y la comunicación emocional e internalización de relaciones. El nivel general de integración estructural es bajo. Los temas conflictivos son reconocibles, pero con frecuencia difíciles de definir claramente en los casos individuales.

Decisivo en la comprensión psicodinámica es el hecho de que las dificultades del paciente no provienen de intenciones inconscientes (deseos, defensas), sino que más bien son consecuencia de una limitada y frágil capacidad de autorregulación y manejo de relaciones, adquirida en el transcurso del desarrollo. De este modo, el 
individuo no se responsabiliza por sus problemas en el sentido de una intención inconsciente. A veces, sin embargo, el paciente muestra un manejo disfuncional de sus limitaciones estructurales, que en sí mismo es problemático (por ejemplo, conductas destructivas) y representa una actividad por la cual puede ser responsabilizado. Por lo tanto, es de fundamental importancia en el contrato terapéutico con estos pacientes distinguir entre sus incapacidades estructurales, por una parte, y las posibilidades de un manejo constructivo o destructivo de ellas. (OPD-2, 2008. p. 393-394).

Cuanto mayor sea la severidad del trastorno estructural, mayor relevancia adquirirán los aspectos del self descritos en el opd-2 (percepción de sí mismo, regulación de sí mismo). En una alteración estructural menos severa, las relaciones objetales adquieren un papel central (percepción del objeto, comunicación): "Ya hemos señalado que en el patrón relacional disfuncional están condensadas todas las disposiciones conflictivas y limitaciones estructurales. Por esta razón, el patrón relacional siempre está en el centro del trabajo terapéutico" (OPD-2, 2008, p. 388) esto es relevante clínicamente porque orienta al terapeuta sobre el énfasis de las intervenciones.

Por último, es necesario tener presente los procesos defensivos en todas las dimensiones estructurales. Las defensas son mecanismos inconscientes utilizados para mantener el equilibrio mental cuando el individuo se enfrenta a tensiones o amenazas tanto internas como externas. La calidad y la efectividad de la defensa son diferentes según el nivel estructural, el progreso en la autonomía del self y el aumento de las capacidades de vínculo. El nivel alto de integración le permite a la persona usar las defensas de manera efectiva, con una distorsión modesta en la prueba de realidad; el nivel bajo de integración y el desintegrado implican un estilo de defensa más primitivo, menos efectivo y una mayor distorsión.

\section{Ilustración clínica del nivel medio de integración}

Un hombre de 30 años, que trabajaba como cajero bancario se postuló a un cargo de gerente regional. Consulta por las dificultades para conciliar el sueño por la noche. Estaba preocupado por el temor de ser humillado "cuando descubran que no tengo aptitudes para ese cargo". Describe un malestar con- 
tinuo en situaciones sociales a causa de su inestable autoestima: "siempre creo que voy hacer algo ridículo". Con frecuencia se imaginaba a sus compañeros de trabajo burlándose de él, de ser ridiculizado en espacios públicos por tener algo indecoroso o feo en su cuerpo. Las imágenes de ser visto como un fracasado han aumentado en las últimas semanas y, con ellas, la ansiedad y la dificultad para conciliar el sueńo.

Recientemente debió asistir a una actividad grupal de bienestar laboral, "hicieron una ronda en la que debíamos expresar las virtudes personales, fue un momento terrible, cuando me llegó el turno no supe qué decir, sudé, me sonrojé, no pude mirarlos y salí de la habitación tan pronto como pude”. El haber ganado la plaza laboral le hizo pensar que había algo positivo en él. Pero rápidamente vuelve a la explicación y origen de sus sentimientos negativos:

Desde niño me sentía raro y me incomodaba estar en juegos o reuniones con mis compañeros. Estaba frecuentemente enojado con sus hermanos porque la madre siempre los prefirió a ellos. También me enojaba conmigo mismo y hablaba de lo malo que era como adelantándome a lo que mi madre podría reclamarme.

Evita hablar de las propias emociones, usa casi la totalidad del tiempo de la entrevista en lo que hace en el trabajo; no tiene vida social ni actividades de recreación.

Las pocas relaciones afectivas con mujeres no han sido duraderas y han estado marcadas por la frustración debido a su patrón relacional inseguro y evitativo. Al respecto, refiere: "No puedo ser yo mismo cuando tengo una relación, me cuesta interpretar las señales afectuosas de mis parejas".

\section{Funciones estructurales más comprometidas en esta viñeta}

La percepción de símismo: el paciente tiene poco interés en reflexionar sobre sí mismo. La autorreflexión se dirige principalmente hacia el selfconductual (lo que el paciente dijo e hizo). Presenta una autoimagen más bien burda.

El Manejo de impulso: la autorregulación se vuelve vulnerable al vivenciar al self como bloqueado o bajo mucha presión y la regulación de la autoestima falla por la alta desvalorización de sí mismo y retiradas. 
Regulación de la relación con el objeto: anticipa reacciones y percepciones exageradas negativas de los otros frente a sí mismo.

Comunicación hacia adentro. Self corporal: es evidente la Inseguridad respecto de la autoimagen, las limitaciones de la vivencia corporal y descripciones egodistónicas

La comunicación de los afectos: es difícil debido a un comportamiento retentivo, recriminatorio y una elevada susceptibilidad.

Su capacidad emocional: se ve disminuida porque la empatía frente al otro se ve limitada bajo la presión de los propios temores.

La capacidad de vínculo: es débil, pues las imágenes internas de personas significativas se diferencian poco.

\section{Conclusiones}

El opD-2 sirve como un marco sólidamente establecido dentro del cual se pueden plantear preguntas sobre procesos dinámicos generales y específicos en la psicopatología de la personalidad debido a su énfasis integrativo de los ejes. Este sistema ofrece perspectivas psicodinámicas complementarias para la comprensión clínica de los problemas de la salud mental de un individuo. Con la orientación diagnóstica de los ejes OPD-2, el terapeuta consigue darle sentido al conjunto de síntomas, vivencias, modos relacionales, conflictos y funcionamiento estructural de un paciente.

La recomendación central es examinar el peso relativo de las limitaciones basadas en conflictos o en la estructura y determinar en qué medida son relevantes para el trastorno del paciente. Esta decisión tiene importantes consecuencias para el tratamiento del paciente, es decir, para la selección del encuadre, de la actitud terapéutica, del foco y de las técnicas terapéuticas.

El terapeuta debe tener una imagen integrada de las interrelaciones e interdependencias dinámicas existentes entre los diferentes aspectos del trastorno identificados por los entrecruzamientos de los ejes y así estar en capacidad de proponer una indicación adaptativa del tratamiento que considera "un traje hecho a la medida" para cada individuo, porque cada formato de intervención conlleva diferentes niveles de exigencia para el paciente respecto a su 
disposición y capacidad (emocional y cognitiva) para la cooperación y trabajo conjunto.

Por tanto, en el campo del diagnóstico estructural, el opD-2 aporta criterios claros y diferenciados de evaluación sobre el nivel dimensional del funcionamiento del self y su relación con los objetos. Los criterios de operacionalización indicados en el manual permiten identificar las vulnerabilidades estructurales, lo que redunda en una indicación del tratamiento útil para el paciente.

\section{Referencias}

American Psychiatric Association (2000). Diagnostic and Statistical Manual of Mental Disorders. Washington, DC: Am. Psychiatr. Assoc. 4th ed., text rev.

Asociación Americana de Psiquiatría (2013). Manual diagnóstico y estadístico de los trastornos mentales (DSM-5). Filadelfia: Asociación Americana de Psiquiatría.

Bender, D., Morey, L. y Skodol, A. (2011). Toward a Model for Assessing Level of Personality Functioning in DSM-5, Part I: A Review of Theory and Methods. Journal of Personality Assessment, 93, 332-346. doi: 10.1080/00223891.2011.583808.

Both, L., Bastos, A. y Freitas, L. (2019) Diagnóstico Psicodinámico Operacionalizado: Una Revisión Sistemática de la Literatura. Tendencias Psiquiatría Psicoterapeuta, 41(2), 201-210.

Cierpka, M., Stasch, M., Grande, T., Schauenburg, H., Parra-Cieciwa, G. de la y Rost, R. (2010). La evaluación primeras entrevistas psicoterapéuticas mediante el sistema diagnóstico psicodinámico operacionalizado (OPD-2). Clínica e Investigación Relacional, 4(1), 221-235.

Cierpka, M., Stasch, M. y Reiner, W. (2006). El sistema Diagnóstico Psicodinámico Operacionalizado (OPD-2): concepto, confiabilidad y validez. Revista chilena de neuro-psiquiatría, 44(2), 105-125. doi: 10.4067/S0717-92272006000200004.

Doering, S., Burgmer, M., Heuft, G., Menke, D., Bäumer, B., Lübking, M., Feldman, M. y Schneider, G. (2014). Assessment of Personality Functioning: Validity of the Operationalized Psychodynamic Diagnosis Axis Iv (Structure). Psychopathology, 47(3), 185-193. doi:10.1159/000355062.

Echevarría-Pérez, R. (2015). El diagnóstico psicopatológico y las clasificaciones de los trastornos mentales. México: Manual de Psicopatología Clínica. 2a ed. 
Trastorno de personalidad: la mirada dimensional del Diagnóstico Psicodinámico Operacionalizado (OPD-2)

Fontenelle, L. y Miguel, E. (2020). The Impact of Coronavirus (COVID-19) in the Diagnosis and Treatment of Obsessive-Compulsive Disorder. Depress Anxiety, 37(6), 510-511.

Fontenelle, L., Oostermeijer, S., Harrison, BJ., Pantelis, C. y Yucel, M. (2011). Trastorno obsesivo-compulsivo, trastornos de control de impulsos y drogadicción. Drugs, 71, 827-840 doi: 10.2165/11591790-000000000-00000.

Gøtzsche-Astrup O. y Moskowitz A. (2016). Personality Disorders and the DSM-5: Scientific and Extra-Scientific Factors in the Maintenance of the Status Quo. Aust NZ J Psychiatry, 50(2), 119-127. doi: 10.1177/0004867415595872.

Grande, T., Dahlbender, R., Schauenburg, H., Stasch, M. y Cierpka, M. (2005) New Diagnostic Alternatives for Dysfunctional Relationships: The Relationship Axis of the OPD-2. Z Psychosom Med Psychother, 51(4), 403-418. doi: 10.13109/ zptm.2005.51.4.403.

Grupo de Trabajo OPD. (2008). Diagnóstico Psicodinámico Operacionalizado (OPD2), Manual para el diagnóstico, indicación y planificación de la psicoterapia. Barcelona: Herder.

Haslam, N., Holland, E. y Kuppens, P. (2012). Categories Versus Dimensions in Personality and Psychopathology: A Quantitative Review of Taxometric Research. Psychol Med, 42(5), 903-920.

Henkel, M., Zimmermann, J., Künecke, J., Remmers, C. y Benecke, C. (2018). Das Potential der Operationalisierten Psychodynamischen Diagnostik für ein differenziertes Verständnis von Persönlichkeitsstörungen [Igual pero diferente: el potencial del diagnóstico psicodinámico operacionalizado para una comprensión diferenciada de los trastornos de la personalidad]. Zeitschrift für Psychiatrie, Psychologie und Psychotherapie, 66(2), 107-117.

Morey, L., Berghuis, H., Bender, D., Verheul, R., Krueger, R. y Skodol, A. (2011). Toward a Model for Assessing Level of Personality Functioning in DSM-5, Part II: Empirical Articulation of a Core Dimension of Personality Pathology. Journal of Personality Assessment, 93(4), 347-353. doi: 10.1080/00223891.2011.577853.

Organización Mundial de la Salud [oms] (2018). Clasificación Internacional de Enfer-

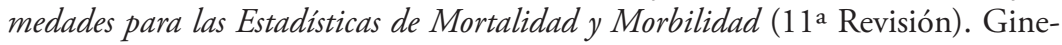
bra: Organización Mundial de la Salud.

Parra-Cieciwa, G. de la, Gómez-Barris, P. y Dagnino, P. (2016). Conflicto y estructura en psicoterapia dinámica: el diagnóstico psicodinámico operacionalizado (opd-2). Mentalización. Revista de Psicoanálisis y Psicoterapia, 6, 1-20. 
Pieh, C., Frisch, M., Meyer, N., Loew, T. y Lahmann, C. (2009) Validity of the Axis "Conflicts" of the Operationalized Psychodynamic Diagnostics (opd-2). Z Psychosom Med Psychother, 55(3), 263-281. doi: 10.13109/zptm.2009.55.3.263.

Pukrop, R., Herpertz, S., Sass, H. y Steinmeyer, E. (1998). Categorical and Dimensional Models of Personality Disorder. Personality and Personality Disorders. A Facet Theoretical Analysis of the Similarity Relationships. Journal of Personality Disorders, 12(3), 226-46.

Rossi-Monti, M. (2012). Sobre las huellas de la vergüenza. El lugar de la vergüenza en psicopatología. Revista Aperturas Psicoanaliticas, 042.

Schneider, G., Mendler, T., Heuft, G. y Burgmer, M. (2008) Validity of Axis III "Conflicts" of Operationalized Psychodynamic Diagnostics (OPD-1). Empirical Results and Conclusions for opd-2. Z Psychosom Med Psychother, 54(1), 46-62. doi: 10.13109/zptm.2008.54.1.46.

Schneider, W., Klauer, T., Freyberger, H., Hake, K. y von Wietersheim, J. (2000) Illness Experience and Treatment Preconditions of Operational Psychodynamic Diagnosis (opd-2). Experiences in Clinical Practice. Psychother Psychosom Med Psychol, 50(12), 454-463. doi: 10.1055/s-2000-9232.

Spitzer, C., Michels-Lucht, F., Siebel, U., Freyberger, H. (2002) On the Validity of the Axis "Structure" of Operationalized Psychodynamic Diagnostics. Z Psychosom Med Psychother, 48(3), 299-312. doi: 10.13109/zptm.2002.48.3.299.

Thomasius, R., Weiler, D., Sack, P., Schindler, A., Gemeinhardt, B., Schuhbert, C. y Küstner, U. (2001) Validity of Operationalized Psychodynamic Diagnostics. Psychother Psychosom Med Psychol, 51(9-10), 365-372. doi: 10.1055/s2001-16896.

Trull, T. y Durrett, C. (2005). Categorical and Dimensional Models of Personality Disorder. Annual Review of Clinical Psychology, 1(1), 355-380. doi: 10.1146/ annurev.clinpsy.1.102803.144009.

Trull T., Distel M. y Carpenter, R. (2011). Dsm-5 Borderline Personality Disorder: At the Border Between a Dimensional and a Categorical View. Curr Psychiatry Rep. 13(1), 43-49. doi: 10.1007/s11920-010-0170-2.

Trull, T., Distel, M. y Carpenter, R. (2010). Dsm-5 Borderline Personality Disorder: At the Border Between a Dimensional and a Categorical View. Current Psychiatry Reports, 13(1), 43-49. doi: 10.1007/s11920-010-0170-2.

Tyrer, P. Reed, G. y Crawford, M. (2015). Classification, Assessment, Prevalence, and Effect of Personality Disorder. Personality Disorder, 385(9969), 717-726. doi: 10.1016/S0140-6736(14)61995-4. 
Vanegas-Osorio, J., Zapata-Patińo, D. y Castrillón-Osorio, L. (2019) Operacionalización psicodinámica del ejeestructura en un paciente drogodependiente. Pensamiento Psicológico, 17(2), 135-147 doi: 10.11144/Javerianacali.PPSI17-2.opee.

Waage, M., Vogel, M., Hake, K. y Schneider W. (2011) The Operationalized Psychodynamic Diagnostics System (opD-2): Validity of the Axis I "Experience of Illness and Prerequisites for Treatment. Psychother Psychosom Med Psychol, 61(34), 154-161. doi: 10.1055/s-0030-1261874.

Zimmermann, J. (2014). Assessing the DSM-5 Level of Personality Functioning and the OPD-2 Level of Structural Integration Amounts to the Same Thing. European Society for the Study of Personality Disorders (ESSPD) Newsletter, 3(1), 9-10.

Zimmermann, J., Ehrenthal, J., Cierpka, M., Schauenburg, H., Doering, S. y Benecke, C. (2012). Assessing the Level of Structural Integration Using Operationalized Psychodynamic Diagnosis (OPD-2): Implications for DSM-5. Journal of Personality Assessment, 94(5), 522-532. doi: 10.1080/00223891.2012.700664. 